\title{
Research Challenges and QoS Provisioning MAC Protocol for Cyber Physical Systems
}

\author{
Saritha I. G., Rajeshwari Hegde
}

\begin{abstract}
One of the latest emerging class of systems which implants cyber features into the physical world is the Cyber Physical System (CPS), which provides a platform for interaction between physical world and virtual world. CPS promises to transform the physical world to virtual world through interaction similar to human interaction with each other. With the increasing demand of cyber physical systems in various applications, it requires wide variety of communication protocols for reliable and real time data transmission. The low- power and low - cost features of some canonical protocols lead to some short falls, reliability and timeliness. In this paper, we discuss an extensive survey on MAC protocols and Research challenges for enhancing the QoS in CPS.
\end{abstract}

Keywords : CPS, MAC and QoS

\section{INTRODUCTION}

It is required to bridge the existing world to the virtual world to accompany the communications and computing with the great revolution from embedded systems to networked CPSs. Cyber physical system is a integration of networking, assessment and physical objects so that networked embedded devices sense, actuate and control the physical world [1]. Though, wireless sensor network became the significantly growing research, research in cyber physical system is moving in the direction to improve the interactions among human and object in the physical world and the virtual world [14]. The QoS linked problems in such networks could be addressed using an efficient QoS aware protocol [12]. Fig. 1 shows the interaction and dataflow between the virtual and physical. In this scenario, the physical world could be the real hospital and the virtual world could be the networked hospital. The interactions happen between these worlds through the intelligent components. The data protection and the security are the biggest challenge in the cyber physical world.

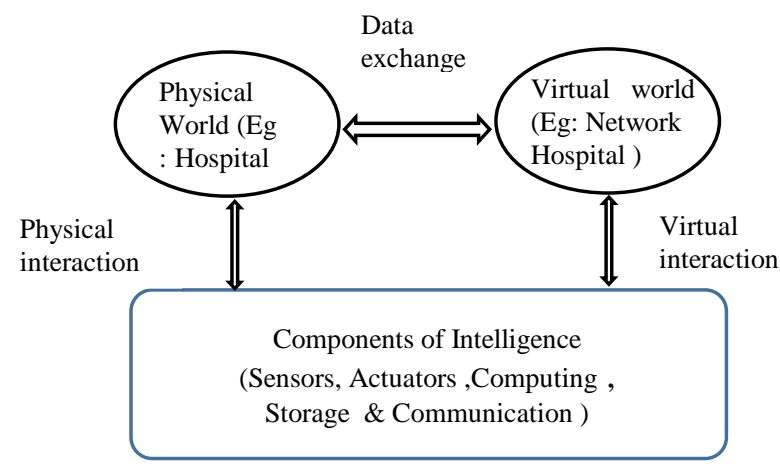

Fig1. Interactions and data flow in CPS

Revised Manuscript Received on December 05, 2019.

Mrs. Saritha I. G., Asst. Professor, Dept. of ETE ,BMSIT, Bengaluru, India.

Rajeshwari Hegde, Dept. of ETE, BMSIT, Bengaluru, India.

\section{Relationship Between Wsn And Cps Features}

1. Quality of services: CPS focuses towards high-level QoS, such as network usage, confidentiality and security of sensing data with high quality of information/intellect etc which is again very important for WSN.

2. Network formation: Network formation in WSN is with low mobility and field-specific which connects on Internet. However, sensor network is dynamic in nature for participation and departure.

3.Communication pattern: WSN incorporates query-response based collective converge communications which allows different routing capability. Some of CPS applications are like flood prevention, water-level controlled dam water gates allows both cross-domain communications and WSN

4. Power management: As sensors are generally deployed on unattended areas, WSN focuses on energy saving with deeper sleeping modes and more redundancy. Whereas activation of sensors have diversified modes in CPS.

5. Network coverage: WSN allows both connectivity and coverage for a single network. The CPS allows different levels of connectivity and coverage for diversified sensor networks.

6. Node mobility: The node mobility is very less in WSN without any control. Whereas the controllable and uncontrollable mobility achieved by CPS applications which lead to collect sensing data from mobile sensor nodes.

7. Knowledge mining: A WSN focuses only on data acquisition whereas CPS emphasizes more on collecting and controlling sensed data and also uses its intelligence to utilize properly.

A good optimized network protocol is a design of an efficient MAC protocol. A controlled and coordinated channel access not only reduces power utilization but also ensures high reliability. This paper is planned as below . Section II meant for the transformation from WSN to CPS. Section III highlighted with the QoS demand in CPS. Section IV deals with related work. Section V gives the Unique Features \& Research challenges in CPS. Section VI deals with CPS handled QoS parameters from MAC protocols. Section VII describes the applications of CPS and is finally concluded in section VIII.

\section{TRANSFORMATION FROM WSNS TO CPS}

By integrating and bridging the gap between information and intelligence of cyber world to sensing and controlling of physical world,

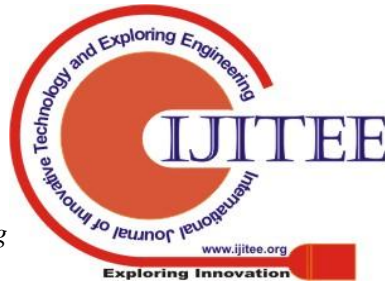


CPS transformed from WSN to overcome the issues like formation of network, power and security. CPS features are categorized in the table 1 .

Table1: CPS applications over Platforms

\begin{tabular}{|c|c|c|c|}
\hline Applications & $\begin{array}{l}\text { Cross domain } \\
\text { Sensor Types }\end{array}$ & $\begin{array}{l}\text { Heterogeneous } \\
\text { Information flows }\end{array}$ & $\begin{array}{l}\text { Intelligent } \\
\text { systems }\end{array}$ \\
\hline $\begin{array}{l}\text { Healthcare } \\
\text { systems }\end{array}$ & $\begin{array}{l}\text { ECG, EEG, } \\
\text { EMG, SPo2, } \\
\text { Accelerometer } \\
\text { and tilt ensors. }\end{array}$ & $\begin{array}{l}\text { Zigbee/BSNs/GPR } \\
\text { S/Bluetooth WLAN }\end{array}$ & $\begin{array}{l}\text { Sensing } \\
\text { entity, } \\
\text { Actuator } \\
\text { entity, Home } \\
\text { manager, } \\
\text { Surveillance } \\
\text { centre, } \\
\text { Locality } \\
\text { Manager, } \\
\text { Local } \\
\text { responder. }\end{array}$ \\
\hline $\begin{array}{l}\text { Navigation \& } \\
\text { Rescue } \\
\text { applications }\end{array}$ & $\begin{array}{l}\text { Smoke, } \\
\text { Temperature, } \\
\text { Humidity, } \\
\text { Camera } \\
\text { sensors, Life } \\
\text { detectors and } \\
\text { Infrared } \\
\text { sensors }\end{array}$ & $\begin{array}{l}\text { Wifi/Zigbee/IEEE8 } \\
02.15 .4\end{array}$ & $\begin{array}{l}\text { Fire Grid } \\
\text {--Data } \\
\text { acquisition } \\
\text {--Simulation } \\
\text { component } \\
\text {--Agent based } \\
\text { Command - } \\
\text { control } \\
\text { component } \\
\text {--Grid } \\
\text { Middleware } \\
\text { component } \\
\end{array}$ \\
\hline $\begin{array}{l}\text { Intelligent } \\
\text { Transport } \\
\text { systems }\end{array}$ & $\begin{array}{l}\text { Accelerometer, } \\
\text { Microphones, } \\
\text { Inertial sensors } \\
\text { and Air sensors, } \\
\text { GPS, } \\
\text { Ultrasonic and } \\
\text { range sensors. }\end{array}$ & $\begin{array}{l}\text { Intra vehicular } \\
\text { communication } \\
\text {-OBD \& CAN bus } \\
\text { Vehicle to vehicle } \\
\text { communication } \\
\text {-DSRC/WAVE } \\
\text { Toll } \\
\text { collection-Electroni } \\
\text { c toll collection } \\
\text { Traffic- Car web }\end{array}$ & $\begin{array}{l}\text { VTrack \& } \\
\text { Park net }\end{array}$ \\
\hline $\begin{array}{l}\text { Social } \\
\text { Networking } \\
\text { and Gaming } \\
\text { Applications }\end{array}$ & $\begin{array}{l}\text { Inertial sensors } \\
\& \text { Thi- chi }\end{array}$ & $\begin{array}{l}\text { BSN/WSn/Wifi } \\
\text { GPS }\end{array}$ & $\begin{array}{l}\text { CenceMe } \\
\text { 1.Conversatio } \\
\text { n classifier } \\
\text { 2.Social } \\
\text { context } \\
\text { 3.Mobility } \\
\text { mode detector } \\
\text { 4.Location } \\
\text { classifier } \\
\text { Am I Hot . }\end{array}$ \\
\hline
\end{tabular}

\section{QOS DEMAND IN CPS}

Need for designing a CPS QoS include

(1) Service-oriented architecture (SOA) lead to decay CPS into various small units as services with quick and scalable growth of CPS.

(2) Cross domain communications to achieve high QoS.

(3) Adaptation and allocation of resources such as Energy, CPU time, Memory and Bandwidth achieved by Minimized resource management.

(4) QoS-aware power management for minimized power requirement [14].

Minimizing resource utilization and maximizing QoS is a great challenge to upcoming CPSs which can be achieved by Cloud computing technique. Cloud computing services categorized as following types:

(1) Infrastructure as a Service assists the lower-layer for users' cloud infrastructures, Platform as a Service (PaaS), facilitated application development by virtual platforms and service APIs and Software as a Service (SaaS) aids a set of software and applications with convenient QoS [14]. Fig. 2 shows the various components of a CPS.

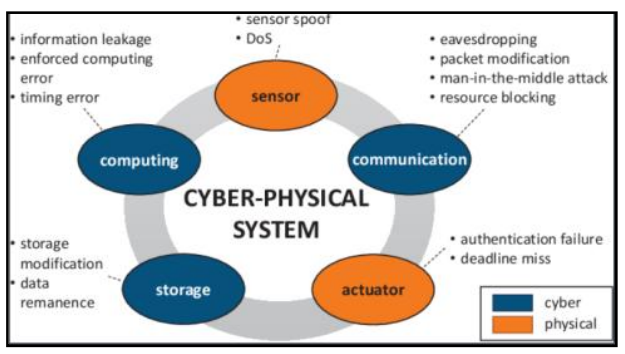

Fig2. Components of CPS

\section{RELATED WORK}

Due to harsh RF environment and applications of wireless networks in CPS which impose hard real time constraints and high reliability differentiates it from the conventional embedded systems.

As MAC protocol is the key consideration for enabling CPS networks, Meng Zheng et al., [5] anticipated a novel MAC protocol to attain instantaneous priority aware polling. In [2], the authors have proposed the optimized design of efficient MAC-RA and a review on the expected challenges and features for IEEE 802.11ax. In the context of TDMA MAC protocols for CPS networks [15] deals with number of slots to allocate for retransmissions. In [11], to achieve low cost channel state estimation, the authors proposed a scheduling algorithm for wireless sensor networks [8] to investigate the scheduling of TDMA slots for retransmissions to reduce the packet drop to avoid the missing of deadlines. In [6], a new protocol for industrial wireless networks was proposed to overcome the limitations of the existing TDMA-MAC protocols.

\section{UNIQUE FEATURES \& RESEARCH CHALLENGES IN CPS}

This section explains the unique features of CPS.

Cross domain sensor exchanges the sensed data with heterogeneous network and intelligent systems.

1. Mobile and Embedded Sensing uses dynamic high degree mobility sensors which vary sensing coverage over time for analyzing mobile data.

2. Give \& take like models to encourage sharing and privacy issues.

3. CPS adopts "pay as you go "concept to serve cloud supported storage, computing and communication capability.

4. Acquired intelligence and comprehension by means of knowledge and data mining is essential for understanding temporal and spatial correlation for sensed data.

5. A rich interaction among many objects such as Sensorsensor, sensor - actuators and actuators- user facilitates various applications through internet of things (IOT).

Following are the reviewed Research challenges and progress for better performance of CPS. 
1. Networking Issues: - Tiny OS based light weighted power saving IP Network architecture, a low powered embedded devices and IPV6 datagram to carry 802.15.4 frames from LoWPAN. IPv6 based network architectures which services with Hop by Hop, Routing protocols and duty cycle . IP link based border router network architecture lead to upcoming CPSs with cross domain peer to peer communication.

2. Cross domain interface avoidance: The difficulty of communication reliability exist due to multiple devices such as Wi-Fi, Bluetooth and Zigbee. To solve the overlapped channels by other networks, a Pessimistic approach is used. A virtual channel approach is used to allow scheduled Zigbee networks by their Super frames. Different channels at diverse period is achieved by Dynamic approach

3. Location based services: Locations of sensor implementation is very important in CPS, as outdoor positioning tracked by GPS, there is a wide demand for indoor positioning which can be scaled up more easily by WLAN infrastructures. Inertial sensors used to facilitate indoor positioning to achieve the signal fluctuation problem.

Small embedded tags used for tracking assets and stolen vehicle.

4. Monitoring services: CPS services support continuous monitoring of both WSN and Cooperative models. The sensor carriers include mobile phones, vehicles and many such devices. Mobieyes is used for urban monitoring by vehicles.

5. Security and Privacy challenges: As sensed data in CPS are not owned by local devices, security and privacy are the two major issues. Determination of degree of privacy achieved by Utilization data collection model for more sensed data acquisition. Perturbation based techniques used for masking the original data by noise which lead to noise cancellation for accurate calculations. Since perturbation technique prone to more errors, the combination of above said two approaches implemented to overcome the same problem. A privacy based vehicular participatory sensing model implemented by linear regression to diminish modeling error and exploit reconstruction error.

\section{CPS HANDLED QOS FROM MAC PROTOCOLS}

\section{A) LPWAN MAC protocols}

LPWANs are newly used for monitoring and controlling delay and bandwidth requirements to achieve reliability. Therefore LPWAN MAC protocols ensure end to end bounded delays. The channel utilization for high data rates of CPS [1] used for Long range communications of order 10's of kms.

LPWAN- MAC protocol is introduced as replacement of LoRA WAN to handle such challenges of CPS to improve bursty traffic patterns and high data rates. LPWAN MAC allows differentiated traffic patterns of data slots and transmission channels to achieve $868 \mathrm{MHz}$ band .

LPWA MAC meet $84 \%$ of peer to peer delays and $100 \%$ of throughput, high reliability and zero collision rate of data packets.

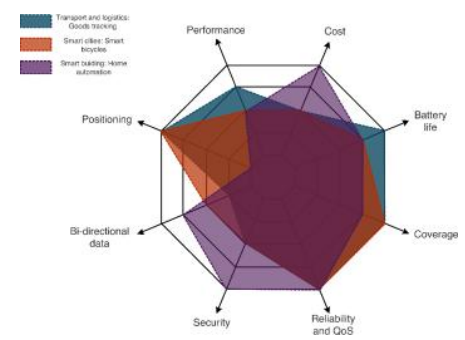

\section{Fig 3 Features of LPWA-MAC DESIGN}

i) Underlying Physical Layer: This LPWA-MAC is easily ported and deliberated to the LoRa physical layers.

ii) Channel Access Scheme: LPWA-MAC make use of central authority (the gateway) to traffic differentiation for its channel access and traffic patterns based data slots and time constrained data. Resources like data slots and transmission channels are allocated on demand, so that LPWA-MAC ensures that effective system reliability and zero data collisions .

iii) Resource Request Discipline: At any time if a node wanted to channel access to transmit data towards the gateway on request, the nodes need to follow the request regulation. A sole channel is reserved for both uplink demand transmissions and downlink demand approval decisions by LPWA-MAC. The gateway performs discrimination of node's traffic to a explicit channel and request based data slot. It also schedules time slots for transmission of node's data so that the node can be in the sleep mode until its slot for sending data to cut down the battery usage.

\section{B) TDMA MAC protocol}

The Industrial wireless sensor network has time constraint in data delivery which is unpredictable due to routing and collision in WSN [4]. To avoid bounded transmission delay and collision of data, TDMA MAC seemed to be very efficient. The slot assignment problem in TDMA is overcome by using simulated anneal algorithm in WSN.

In WSN, the environment status sensed and sends data periodically to sink node which forwards same data to control the centre which take right action to respond. A signal collision problem may take place since data delivery in WSN along with CSMA/CA MAC protocol will lead to raise an issue of reaching sensed data to control centre in bounded delay. To overcome this problem, TDMA MAC protocol is used to have bounded transmission delay so that in every time slot single sensor node is allowed to transmit which achieves collision free medium access using slot assignment scheme with spatial reuse and data aggregation for minimizing the time required for sensed data acquisition and longer the system operation under limited battery power to improve the energy efficiency.

\section{SLOT ASSIGNMENT ALGORITHM}

a) Topology Construction: The Dijkstra algorithm is used for deriving routing path from every sensor node to sink node. The sensor node sends collected data from neighbor node to sink node and again it replies back 
with acknowledgement frame which includes identity of a node its MAC address and number of hops to the source node. The sensor node again sends the neighbor node table to the sink node if sensor node doesn't receive any neighbor discover frame. Once the sink node collects data from all neighboring node table sent by the sensor nodes, the topology is constructed.

b) TDMA Slot Assignment: TDMA MAC uses a frame which is necessary for assigning slots for all sensor nodes, which allocates one slot to one sensor node, when sensor node overlap each other, these sensor nodes can be allocated to identical slot to reduce frame length.

c) Adaptive MAC protocol: With the rising demands for healthcare systems, Cyber physical systems emerged as high-quality health care monitoring system over Wireless body sensor networks [7]. Communication protocols are required to achieve both reliable, real-time data transmissions with zero collision and more adaptation. IEEE 802.15.4 is a limited power and minimum cost protocols lead to achieve low reliability. Whereas IEEE 802.15.4, based Adaptive MAC protocol is a cross protocol which combines both scheduled based time triggered and contention based protocols with guaranteed time slots allocation. Fig. 4 shows the application of CPS in medical field. The physical parameters from patients are collected using different sensors and the report is sent to the doctor for diagnosis.

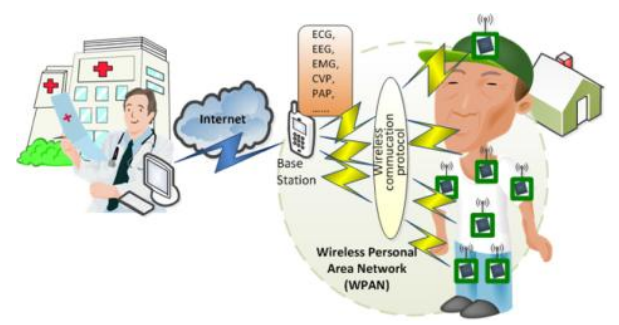

Fig 4. Application of CPS in Medical field

\section{(a)IEEE 802.15.4 and time-triggered protocol}

IEEE 802.15.4 is a typical protocol intended for both Physical layer and MAC layer of a small-rate wireless network namely PAN. The Physical layer includes 27 communication channels and acts in three different frequency bands. The MAC layer uses two different modes: beacon-enable mode which enable beacon frame, a time between two consecutive frames to transmit periodically and non beacon-enable which disables beacon frame. The superframe divides equally into sixteen time slots; it contains beacon frame, a contention access period (CAP) and a contention free period (CFP). The Contention free period is elective and contains up to seven GTSs (Guaranteed Time Slot) in every superframe is stored for the particular nodes to transmit time considerable packets.

Time-triggered protocol: This is used in scattered real-time applications which needed a high reliability and it avoids collisions. It also guarantees the data transmission with expected little latency and serviced with minimal overhead.

Superframe structure : This super frame is categorized into a number of fixed mini-slots and each one is long enough to transmit each data packet. The super frame in turn subdivided into the following three periods: (1) Contention free period consists a number of PAN coordinator allocated GTS to the particular nodes to transfer real-time data. (2) CAP is located after the CFP so that every node can send ordinary data using the slotted based CSMA/CA mechanism during the CAP.

(3) The inactive period

D) Adaptive CSMA/CA

CPS provisioned QoS in Wireless sensor / actual network plays an important part in applications such as Smart grid, which uses IEEE 802.15.4 in providing multiple QoS [10]. IEEE 802.15.4 slotted CSMA/CA provide static services without providing differentiated services and self adaptability in networks which was overcome by Priority based service differentiated \& Adaptive CSMA/CA algorithm to provide differentiated QoS such as Effective data rate, Average delay and Packet loss rate. In Service differentiation Mechanism, service differentiation is needed to enable devices that produce emergency data to take priority over other devices. It consists of three levels $\mathrm{L} 1<\mathrm{L} 2<\mathrm{L} 3$ for varying different parameters such as Contention Windows, mac Min BEs and mac Max BEs according to priority of the devices.

In Adaptive Backoff mechanisms, it can adaptively adjust BE to traffic conditions. When there is a heavy traffic, a larger BE is used while a smaller BE is used for light traffic, which leads to transmit more effective data packets with less waiting period and smaller amount of collisions. This shows that the future traffic load could be estimated by the effective data rate.

Some of the QoS parameters of CPS in IEEE802.15.4 are listed below

a. Average delay-It is the ratio of average time required by data packet to successful packet reception. It is used to evaluate the performance of a real time network.

$$
\begin{aligned}
& \mathrm{D}_{\text {avg }}=\frac{\text { Navg }}{\text { Nsusspacket }}= \\
& \Sigma \frac{\text { (Total delay by all packets from start to end of reception) }}{(\text { Total number of packets successfully received by all nodes) }}
\end{aligned}
$$

b. Effective data rate-This parameter is used to assess link between CPS resource consumption and network dependability.

$$
\mathrm{R}_{\text {eff Data }}=\frac{N \text { susspacket } x \text { LMSDU }}{\text { Tend }- \text { Tstart }}
$$

Where $\quad \mathrm{L}_{\mathrm{MSDU}}=\mathrm{MSDU}$ length of data frames and $\mathrm{T}_{\text {end }}-\mathrm{T}_{\mathrm{start}}=$ Total time required for transmission

c. Packet Loss rate- This is the ratio of total traffic dropped by network to overall traffic which shows the degree of reliability for effective transmission in CPS.

$$
\mathrm{R}_{\text {loss }}=\frac{N \text { dropped }}{\text { Ngenerated }}=\frac{\text { Traffic dropped by network }}{\text { Overall traffic }}
$$

\section{E) PLA MAC(Priority and Load adaptive MAC) protocol}

Priority and load adaptive MAC protocol for BSNs maintains efficiency in power consumption to improve their QoS and Transmission of data packets schedules based on their priorities. Super frame structure of PLA MAC protocol varies depending on power consumption and quantity of traffic load BSNs are emerging CPS through improved, lowcost healthcare systems by incorporating improved sensing and actuations [9]. 
Data packets of PLA-MAC for BSN based on their priority and dynamic super frame structure where slots allocation in the structure vary based on the traffic load .Superframe structure consists of inactive period during low traffic load and it is active when traffic load is high with energy efficiency.

PAL MAC protocol Modifies superframe structure of IEEE 802.15.4 in BSN , traffic classification is based on delay and reliability constraints of data packets .

The different priority and back off calculated by classification and data generated by sensor nodes, where as a coordinator uses priority for allocation of slots for data packets and sensor nodes uses back off to perform priority based random back off for data packet transmission .

Traffic classifications-Data packets are categorized as follows in PAL-MAC

a)Ordinary data packets (OPs) measures physical characteristics such as body temperature but does not have reliability or dealy constraints .

b) Delay driven data packets (DPs) delivered timely without much reliability Ex: Video streaming .

c) Relaibility driven data packets (RPs) delivers a packet with high reliability Ex:Both respiration and ph monitoring. d)Critical data packets (CPs) delivered with high relaibility and any delay deadline Ex: ECG data.

Superframe structure: The five periods of frame structure are firstly, Beacon frame allows super frame structure to start and gives basic information about coordinator and nodes to all the member. Secondly, Contention Access Period (CAP) allows allocation requests for $\mathrm{CP}, \mathrm{RP}$ and $\mathrm{OP}$ packets in Contention free period(CFP). CFP can be very small in low traffic and is high in high traffic load, so that it occupies rest of superframe. Otherwise CFP does not use superframe which will be inactive period.

Backoff Calculation : Each node performs a random Backoff, which transmits either packet data or acknowledgement packet in CAP. Backoff is calculated based on value of traffic class. The packet data with lesser traffic have small back off value and high traffic class have large Backoff value .

Priority calculation : Priority of each packet calculated by sensor nodes $\mathrm{P}_{\mathrm{i}}=\frac{T \mathrm{i}}{\text { Gi } * S i}$

Where $\mathrm{P}_{\mathrm{i}}=$ Priority, $\mathrm{T}_{\mathrm{i}}=$ Traffic class value and $\mathrm{S}_{\mathrm{i}}=$ Size in bytes, $\mathrm{G}_{\mathrm{i}}=$ Data generation rate.

PLA - MAC significantly improves the following QOS metric for performance evaluation

- Average Packet delivery delay - It is time between packet generation at sensor node and reception at sink node in definite slot of cooresponding superframe.

- Average Packet delivery delay for delay driven packet - Thease pasckets are to be delivered in deadline so that delay reduces .

- Throughput - The average rate of successful delivery of packets over a comunication channel over a unit time is measured by throughput which increases with the number of nodes.

- Coordinator Power requirement - The power utilized at different states such as transmit, receive, listening and sleep is different. IEEE 802.15.4 allows low power cosumption due to long inactive period. PLA MAC power consumption is low for low traffic and it increases linearly with traffic load.

\section{RESULTS AND DISCUSSIONS}

1. Smart Building: The building control application of CPS can prevent logical and physical as well as external and internal attacks. The substantial integration of the building is implemented with power, transportation, first response and law enforcement.

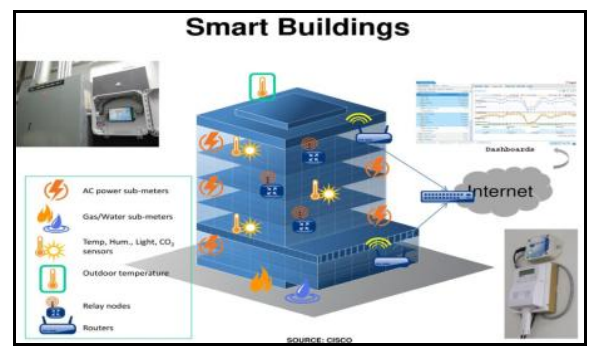

Fig5.Smart building application in CPS

\section{Agriculture}

Due to exponential increase in the worldwide population, there is a high risk of food shortage due to unstable climate change. As per the survey, today, more than $50 \%$ of food is wasted during production, storage and transportation. Smart agriculture system using CPS could be employed to reduce the food wastage. The use of CPS system will improve the processing of food at various stages using smart sensors and actuators for collecting and processing intelligent data from agriculture systems there by increasing the operational efficiency and reduction of pollution.

\section{Cyber Defense}

The CPS cyber security over a network systems play a critical role for achieving military missions and national defense requirements. The flexibility, robustness and resilience would be ensure by the software enabled CPS. To provide situational awareness to the public during disaster, the intelligent systems can alert the public well in advance using CPS enabled early warning systems, public safety systems and other such applications.

\section{Smart Manufacturing}

The CPS with IoT covers protocols, applications, and other domains for smart manufacturing. It is the web that not only enables machine-to-machine communication (M2M) but also hooks up the physical objects with virtual intelligence and services. The physical devices would gather the data and virtual system can process and exchange the information.

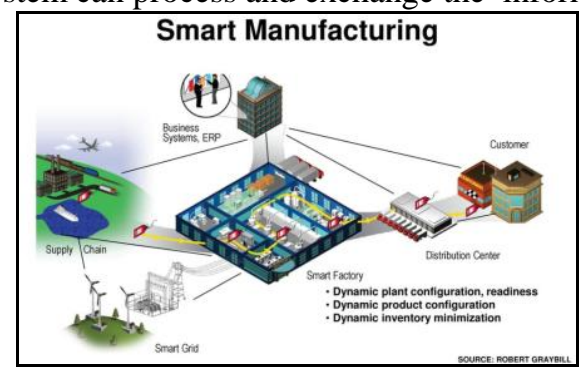

Fig 6. Smart Manufacturing application in CPS
Blue Eyes Intelligence Engineering \& Sciences Publication 


\section{Research Challenges and QoS Provisioning MAC Protocol for Cyber Physical Systems}

\section{VIII.CONCLUSION}

The Cyber Physical System is a future class of research area which provides wide opportunities for the technologists to explore in many different fields. It is a multidimensional system that integrates both cyber word and the physical world, raised to have increasing interest towards privacy for control systems with differential privacy, such as data minimization by changing sampling period and homo-morphic cryptography in feedback systems. The future application of CPS in terms of energy, transportation, robotics fields and healthcare systems with wearable electronic devices or sensors. Through the approach of Cyber-physical system, the human get closer to nature which results in reduced casualties, calamities and material loss with future trend research such as creation of tools to speed up the system development process.

\section{REFERENCES}

[1] Laksh Bhatia, Julie A. , McCann and Ivana Tomić "Poster Abstract: LPWA-MAC - a Low Power Wide Area network MAC protocol for cyber-physical systems"2018. Cornell university.

[2] Rashid Ali 1, Sung Won Kim "Design of MAC Layer Resource Allocation Schemes for IEEE 802.11ax: Future Directions" 1,2, Byung-Seo Kim 2 and Yongwan Park1IETE TECHNICAL REVIEW, 2018

[3] Zongyi Liu!, Daniela Dragomirescu†, Georges Da Costa! and ThierryMontei "Dynamic Multi-Channel Allocation Mechanism for Wireless Multimedia Sensor Networks" $1 \uparrow$ Open Archive TOULOUSE Archive Ouverte (OATAO) WD 2016.

[4] Ching-Lung Chang , Kuan-Yi Ho, "Slot Assignment for TDMA MAC in Industrial Wireless Sensor Network" IEEE ICIS 2016

[5] Meng Zheng, Wei Liang, Junru Lin and Haibin Yu "A Priority-aware Frequency Domain Polling MAC Protocol for OFDMA-based Networks in Cyber-physical Systems" IEEE/CAA Journal of Automatica Sinica, Vol. 2, No. 4, 2015

[6] Xiao Y, Lin J, Liang W, Yu H B. Polling in the frequency domain: a new MAC protocol for industrial wireless network for factory automation. International Journal of Ad Hoc and Ubiquitous Computing, 2014 http://www.inderscience.com/info/ingeneral/

[7] Feng Xia, Linqiang Wang, Daqiang Zhang, Daojing He, Xiangjie Kong "An adaptive MAC protocol for real-time and reliable communications in medical cyber-physical systems" Published online: 10 December 2014 () Springer Science+Business Media New York 2014.

[8] Willig A, Uhlemann E.” Deadline-aware scheduling of cooperative relayers in TDMA-based wireless industrial networks. Wireless Networks", 2014, 20(1): $73 ; 88$

[9] Iffat Anjum, Nazia Alam, Md. Abdur Razzaque, MohammadMehedi Hassan, and Atif Alamri, "Traffic Priority and Load Adaptive MAC Protocol for QoS Provisioning in Body Sensor Networks “.International Journal of Distributed Sensor Networks, Hindawi Publishing Corporation Vol : 2013.

[10] Feng Xia, Jie Li, Ruonan Hao, Xiangjie Kong, and Ruixia Gao "Service Differentiated and Adaptive CSMA/CA over IEEE 802.15.4 for Cyber-Physical Systems" Hindawi Publishing Corporation The Scientific World Journal Volume 2013.

[11] Zhang X L, Liang W, Yu H B, Feng X S." Reliable transmission scheduling for multi-channel wireless sensor networks with low-cost channel estimation. IET Communications", 2013, 7(1): 71;81

[12] Anup Thapa and Seokjoo Shin "QoS Provisioning in Wireless Body Area Networks: A Review on MAC Aspects" KSII Transactions on Internet And Information Systems Vol. 6, No. 5, 2012

[13] Feng Xia,1 Alexey Vinel,2, 3 Ruixia Gao,1 LinqiangWang,1 and Tie Qiu1 "Evaluating IEEE 802.15.4 for Cyber-Physical Systems" Hindawi Publishing Corporation EURASIP Journal on Wireless Communications and Networking Volume, 2011
[14] Fang-Jing Wua, Yu-Chee Tseng a. Yu-Fen Kaob, "From wireless sensor networks towards cyber physical systems Science Direct (C) 2011 Elsevier B.V.

[15] Zheng M, Liang W, Yu H B. "An optimization framework for optimal replicator factors control in wireless sensor network"s. Adhoc and Sensor Wireless Networks, 2011, 13(3;4): $271 ; 289$

\section{AUTHORS PROFILE}

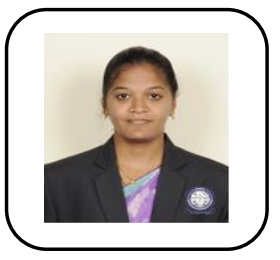

Mrs. Saritha I. G. Completed B.E (TCE), from MVIT, Bangalore, M.Tech (Digital electronics and communication) from MSRIT Bengaluru, and Pursuing Ph.D (Cyber security) from Visvesvaraya University, Belagavi. she has 12 years of teaching, 3 years of research experience. At Present, working as Asst.Professor, ETE Dept.,BMSIT, Bengaluru. Member for professional bodies, ISTE \& IAPURAI. Organized and attended many Workshops, FDPs and STTPs. Guided UG Projects Published 11 Technical papers in National \ International journals \ Conference

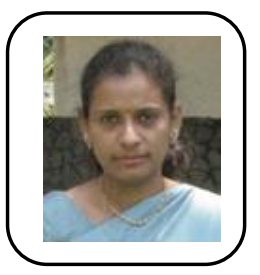

Rajeshwari Hegde received her Bachelor of Engineering in Electronics and Communication Engineering from National Institute of Engineering, Mysore, Master of Engineering in Electronics from BMS College of Engineering, Bangalore and PhD from Bangalore University. She is presently associated with the Department of Electronics and Telecommunication Engineering of BMS College of Engineering, Bangalore, India. Her Research interests include embedded systems and Communication. She has published 120 research papers in reputed journals and conference. 